\title{
PERFIL DEMOGRÁFICO E CLÍNICO DE PORTADORES DE DOENÇA PULMONAR OBSTRUTIVA CRÔNICA NO SUL DO BRASIL
}

\author{
DEMOGRAPHIC AND CLINICAL PROFILE OF PATIENTS OF CHRONIC \\ OBSTRUCTIVE PULMONARY DISEASE IN SOUTHERN BRAZIL
}

\section{PERFIL DEMOGRÁFICO Y CLÍNICO DE PACIENTES PORTADORES DE ENFERMEDAD PULMONAR OBSTRUCTIVA CRÓNICA EN EL SUR DE BRASIL}

\author{
Cássia Cinara da Costa ${ }^{*}$ \\ Daiane Bolzan Berlese ${ }^{* *}$ \\ Rafael Machado de SOUZA ${ }^{* * *}$ \\ SAMANTA Siebel ${ }^{* * * *}$ \\ Paulo José Zimermann Teixeira ${ }^{* * * *}$
}

\section{RESUMO}

Objetivo: Caracterizar uma população de pacientes portadores de Doença Pulmonar Obstrutiva Crônica (DPOC) acompanhados em um Programa de Reabilitação Pulmonar (PRP). Método: O estudo foi observacional descritivo, com 83 pacientes com diagnóstico de DPOC. Todos os pacientes realizaram espirometria e exames laboratoriais bioquímicos. Resultados: Foram incluídos 47 homens (56,6\%) e 36 mulheres (43,4\%). A comorbidades Hipertensão Arterial (HAS) foi mais relevante em 21 (44,6\%) dos homens e 14 (38,8\%) das mulheres, seguido de cardiopatias 8 homens (17\%) e 2 mulheres (5,5\%) e Diabetes Mellitus (DM), sendo esta uma comorbidade encontrada quase que exclusivamente em homens, 7 (14,8\%) e apenas 1 mulher $(2,7 \%)$. Conclusão: $\mathrm{O}$ acampamento dos parâmetros clínicos e laboratoriais de forma sistemática pode auxiliar na promoção e prevenção das comorbidades associadas a doenças.

Palavras chave: Doença pulmonar obstrutiva crônica, epidemiologia, centro de reabilitação pulmonar.

\footnotetext{
ABSTRACT

Objective: To characterize a population of Chronic Obstructive Pulmonary Disease (COPD) patients followed through a Pulmonary Rehabilitation Program (PRP). Methods: the study was observational, descriptive, with 83 patients with a diagnosis of COPD. All patients underwent spirometry and biochemical laboratory tests. Results: 47 men (56.6\%) and 36 women (43.4\%) were included. The most relevant comorbidity was Hyperten-

* Fisioterapeuta, Doutora em Ciências Pneumológicas. Professora do curso de fisioterapia da Universidade Feevale. Novo Hamburgo, Brasil. E-mail: cassiac@feevale.br

${ }^{* *}$ Química, Doutora em Bioquímica Toxicológica. Professora do Instituto de Ciências da Saúde da Universidade Feevale. Novo Hamburgo, Brasil. E-mail: daianeb@feevale.br

${ }^{* * *}$ Educador Físico, Mestrado em Qualidade Ambiental. Professor do Instituto de Ciências da Saúde da Universidade Feevale. Novo Hamburgo, Brasil. E-mail: rafaelms@feevale.br

${ }_{* * * *}$ Enfermeira, aluna de Aperfeiçoamento Científico da Universidade Feevale. Novo Hamburgo, Brasil. E-mail: samynh@ hotmail.com

${ }^{* * * * *}$ Médico, Doutor em Medicina. Professor do Instituto de Ciências da Saúde da Universidade Feevale. Novo Hamburgo, Brasil.E-mail: paulojzt@feevale.br
} 
sion, present 21 men (44.6\%) and 14 woman (38.8\%), followed by heart disease: 8 men (17\%) and two women (5.5\%) and Diabetes Mellitus (DM), a comorbidity found almost exclusively in men $7(14.8 \%)$ and only 1 woman $(2.7 \%)$. Conclusions: The systematic monitoring of the clinical and laboratory parameters can help in the promotion and prevention of comorbidities associated with diseases.

Key words: Chronic obstructive pulmonary disease, epidemiology, pulmonary rehabilitation center.

\section{RESUMEN}

Objetivo: Caracterizar una población de pacientes con Enfermedad Pulmonar Obstructiva Crónica (EPOC) seguidos en un programa de rehabilitación pulmonar (PRP). Material y métodos: El estudio fue observacional descriptivo, con 83 pacientes diagnosticados de EPOC. Todos los pacientes fueron sometidos a espirometría y a pruebas de laboratorio bioquímicas. Resultados: Se incluyeron 47 hombres $(56,6 \%)$ y 36 mujeres (43,4\%). La hipertensión fue la comorbilidad más relevante en 21 hombres $(44,6 \%)$ y 14 mujeres $(38,8 \%)$, seguida de las enfermedades del corazón 8 hombres (17\%) y dos mujeres (5,5\%) y Diabetes mellitus (DM), que es una comorbilidad que se encuentra casi exclusivamente en hombres, 7 (14,8\%), y sólo en 1 mujer (2,7\%). Conclusión: El seguimiento de los parámetros clínicos y de laboratorio de manera sistemática puede ayudar en la promoción y prevención de las comorbilidades asociadas a enfermedades.

Palabras clave: Enfermedad pulmonar obstructiva crónica, epidemiología, centro de rehabilitación pulmonar.

Fecha recepción: 30/03/15 Fecha aceptación: 10/08/16

\section{INTRODUÇÃO}

A Doença Pulmonar Obstrutiva Crônica (DPOC) é caracterizada pela limitação crônica do fluxo aéreo. A limitação do fluxo aéreo geralmente é progressiva e associada a uma resposta inflamatória anormal do pulmão, a partículas ou gases nocivos. Exacerbações e comorbidades contribuem para a severidade da doença individualmente (1). Atualmente é a quarta causa de morte no mundo, representando um importante problema de saúde pública, desafio que é prevenível e tratável. Sendo a maior causa de mortalidade e morbidade crônica em todo o mundo, muitas pessoas sofrem desta doença por anos e morrem prematuramente por esta ou por suas complicações. Globalmente a DPOC esta projetada para aumentar, pois as pessoas continuam expostas a fatores de risco, sendo o tabagismo o principal, além de exposição a fogão a lenha, poluição ambiental, fatores genéticos e desnutrição (1). No Brasil, a DPOC e a terceira causa de morte entre as doenças crônicas não transmissíveis, com um aumento de $12 \%$ no número de óbitos entre 2005 e 2010, o que representa atualmente quase 40.000 óbitos anuais decorrentes da DPOC (2).

Além do comprometimento pulmonar a DPOC apresenta manifestações sistêmicas relacionadas, como: intolerância ao exercício físico, disfunção muscular periférica, alterações nutricionais e exacerbações recorrentes levando a hospitalizações (3). A perda de massa muscular, alterações nas fibras musculares e no fluxo sanguíneo, além de acidose láctica precoce, contribuem para a intolerância ao exercício físico (4). Além destes fatores, a dispneia é o principal sintoma desta doença, onde os pacientes tem uma redução das atividades de vida diária (AVD's), desencadeando assim o ciclo vicioso da dispneia, onde os pacientes cada vez fazem menos atividades e sentem mais falta de $\operatorname{ar}(1,5,6)$.

O diagnostico da DPOC é confirmado através da espirometria pela razão do volume expiratório forçado no primeiro segundo $\left(\mathrm{VEF}_{1}\right)$ pela capacidade vital forçada 
(CVF) inferior a $70 \%$ do previsto obtido pela espirometria, além da história clínica dos pacientes (7). Porém sabe-se que além da alteração pulmonar existem outras variáveis importantes para o prognostico destes pacientes, bem como: o índice de massa corpórea (IMC), a exposição ou não ao tabaco. As comorbidades ocorrem frequentemente em portadores de DPOC incluindo: doenças cardiovasculares, disfunções musculoesqueléticas, síndrome metabólica, osteoporose, depressão e câncer pulmonar, tendo uma grande importância na evolução e tratamento destes pacientes. Assim os exames bioquímicos ou laboratoriais tornam-se um aliado importante para o diagnóstico de algumas comorbidades (8-11).

Assim, conhecer o perfil desses pacientes com DPOC torna-se fundamental na prevenção de comorbidades associadas a doença respiratória. Para tanto, com a realização do estudo, espera-se contribuir para a ampliação das discussões sobre essa problemática e as comorbidades associadas que impõe ao paciente a limitação física e o aumento das taxas de mortalidade, bem como colaborar para a construção de uma prática que fortaleça a abordagem multidisciplinar e interdisciplinar frente ao tratamento de pacientes com DPOC.

Portanto, o objetivo deste estudo foi de identificar o perfil demográfico, bioquímico e hematológico de uma população de pacientes portadores de DPOC acompanhados em um Programa de Reabilitação Pulmonar na Universidade Feevale, Sul do Brasil.

\section{MATERIAL E MÉTODO}

Tipo de estuda e amostra: Estudo observacional descritivo, de correlação, com uma amostra consecutiva de 83 pacientes com diagnóstico de DPOC segundo critérios definidos GOLD (7) que participaram de um Programa de Reabilitação Pulmonar da Universida- de Feevale na cidade de Novo Hamburgo/RS. Coleta de dados: Os dados foram coletados por profissionais da área da saúde, médico pneumologista, fisioterapeutas e bioquímicos. Os instrumentos utilizados para a coleta de dados foram: história clínica, exame físico e a confirmação da obstrução do fluxo aéreo através da razão do volume expiratório forçado no primeiro segundo $\left(\mathrm{VEF}_{1}\right)$ pela capacidade vital forçada (CVF) inferior a 70\% do previsto obtido pela espirometria. Todos os pacientes apresentavam DPOC de moderado a grave, definido por um $\mathrm{VEF}_{1}<60 \%$ do valor previsto após o uso de broncodilatador (7). Função pulmonar foi medida pelo teste de espirometria, onde o paciente foi orientado a ficar sentado com as pernas relaxadas e os pés no chão com o clip nasal a inspiração até a capacidade pulmonar total (CPT) antes da expiração forçada não deve ser muita rápida. A pausa pós inspiração não deve exceder 3 seg, o número de tentativas deve levar em conta 3 curvas aceitáveis e duas reprodutíveis com no máximo 8 repetições, espirometro utilizado foi o Microlab ML-3500. Todos os pacientes participaram do PRP da Universidade Feevale no período de março de 2007 a dezembro de 2012.

Os pacientes responderam um questionário padronizado com informações sobre: sexo, idade, peso, índice de massa corporal, histórico de tabagismo (Pack years), comorbidades, além das variáveis obtidas da espirometria. Para avaliação antropométrica da massa corporal e da estatura, foi utilizada uma balança da marca Walmy, com precisão de $100 \mathrm{~g}$, e um estadiômetro fixo da marca Cardiomed, com precisão de $1 \mathrm{~mm}$. O histórico de tabagismo (Pack years) foi obtido pelo cálculo onde se multiplica o número de maços de cigarro consumido pelo paciente por ano, então se obtém o pack years ou seja o número de maços fumados por ano.

O PRP tem duração de 12 semanas, ocorreu segunda, quarta e sexta-feira no turno da manhã. As sessões duravam em média duas horas, divididas em atividades físicas e 
palestras educacionais. Consistia em aquecimento, exercícios físicos aeróbicos com a utilização de esteira ergométrica, exercícios de fortalecimento de membros superiores, membros inferiores e alongamentos.

Após o período de reabilitação os pacientes foram encaminhados ao laboratório de Biomedicina da Universidade para realizar os exames laboratoriais, foram realizados exames bioquímicos (glicemia, colesterol total, colesterol HDL, colesterol LDL, triglicerídeos, ureia, albumina e creatinina) e hematológicos (Hemoglobina, VCM, Eritrócitos, Leucócitos), para a realização destes exames os pacientes foram orientados a permanecer $12 \mathrm{~h} \mathrm{em} \mathrm{jejum.} \mathrm{Todos} \mathrm{os} \mathrm{pacientes} \mathrm{assinaram}$ o termo de consentimento livre e esclarecido. A pesquisa foi iniciada após a aprovação pelo comitê de ética da Universidade Feevale, processo número 4.08.03.08.1239.

Análise de dados: As variáveis da caracterização da amostra foram representadas por estatística descritiva utilizando média e erro padrão da média, além da utilização do teste t de Student.

\section{RESULTADOS}

Foram incluídos 83 pacientes portadores de DPOC, 47 homens $(56,6 \%)$ e 36 mulheres $(43,4 \%)$ com idade média de 65,85 para homens e 65,16 para mulheres. Em relação ao hábito tabágico todos os homens eram ex-fumantes e $6(16,6 \%)$ as mulheres nunca fumaram. A HAS foi a comorbidade mais relevante em $21(44,6 \%)$ dos homens e $14(38,8 \%)$ das mulheres seguido de cardiopatias $8(17 \%)$ dos homens e das $2(5,5 \%)$ mulheres. O DM foi encontrado quase que exclusivamente em homens $7(14,8 \%)$ e apenas 1 mulher $(2,7 \%)$ (Tabela 1).

Tabela 1. Características antropométricas e clínicas dos 83 pacientes DPOC participantes de um programa de reabilitação pulmonar.

\begin{tabular}{lcc}
\hline & \multicolumn{2}{c}{ Sexo } \\
\cline { 2 - 3 } Variáveis & Masculino & Feminino \\
\cline { 2 - 3 } & Média $( \pm$ EPM $)$ & Média $( \pm$ EPM $)$ \\
\hline Idade (anos) & $65,85( \pm 1,2)$ & $65,16( \pm 1,5)$ \\
Peso $(\mathrm{kg})$ & $74,40( \pm 2,1)$ & $60,45( \pm 1,6)$ \\
Altura $(\mathrm{m})$ & $1,68( \pm 0,0)$ & $1,55( \pm 0,0)$ \\
IMC $\left(\mathrm{kg} / \mathrm{m}^{2}\right)$ & 22,9 & 25,1 \\
VEF 1 L & 1,5 & 1 \\
CVF L & 2,6 & 1,7 \\
VEF $/$ /CVF & 50,2 & 58,6 \\
Nunca Fumaram & 0 & 6 \\
Pack Years & $76,1( \pm 2,2)$ & $41,2( \pm 0,9)$ \\
Comorbidades & & $14(38,8 \%)$ \\
HAS & $21(44,6 \%)$ & $1(2,7 \%)$ \\
DM & $7(14,8 \%)$ & $2(5,5 \%)$ \\
Cardiopatias & $8(17 \%)$ &
\end{tabular}

$\mathrm{EPM}=$ erro padrão da média; $\mathrm{kg}=\mathrm{kilo} ; \mathrm{m}=$ metro; $\mathrm{IMC}\left(\mathrm{kg} / \mathrm{m}^{2}\right)=$ índice de massa corporal (kilo/metro quadrado); $\mathrm{VEF}_{1} \mathrm{~L}=$ volume expiratório forçada no primeiro segundo em litros; $\mathrm{CVF} \mathrm{L}=$ capacidade vital forçada em litros (percentual do predito); $\mathrm{VEF}_{1} / \mathrm{CVF}=$ volume expiratório forçada no primeiro segundo em litros (percentual do predito)/ capacidade vital forçada em litros (percentual do predito); HAS: hipertensão arterial; DM: diabete mellitus 
Outro aspecto importante a ser destacado no estudo são pacientes com mais de uma comorbidades, $3(6,4 \%)$ dos homens apresentaram mais de uma e $4(8,5 \%)$ com as três comorbidades pesquisadas, enquanto nas mulheres apenas $1(2,7 \%)$ paciente com duas.

Os resultados dos exames laboratoriais hematológicos estão apresentados na Tabela 2. Os valores estão apresentados como mé- dias para homens e mulheres respectivamente. Hemoglobina de 14,5 e 13,7 (g/100mL), estes valores diferem significativamente entre homens e mulheres. O hematócrito corresponde a 43,9 e 42,1\%, o Volume corpuscular médio (VCM) foi de 90,4 e $90\left(\mu^{3}\right)$, os eritrócitos estavam entre os valores de 4,9 e $4,7\left(\mathrm{x} 10^{6} / \mathrm{mm}^{3}\right)$ e os Leucócitos foram 7556 e $7095\left(\mathrm{~mm}^{3}\right)$.

Tabela 2. Comparação do hemograma, por sexo, de pacientes com DPOC participantes de um programa de reabilitação pulmonar.

\begin{tabular}{lcc}
\hline \multirow{2}{*}{ Variáveis } & \multicolumn{2}{c}{ Sexo } \\
\cline { 2 - 3 } & Masculino & Feminino \\
\cline { 2 - 3 } & Média $( \pm \mathrm{EPM})$ & Média $( \pm \mathrm{EPM})$ \\
\hline Hemoglobina $(\mathrm{g} / 100 \mathrm{ml})$ & $14,5( \pm 0,2)^{\star}$ & $13,7( \pm 0,1)$ \\
Hematócrito $\%)$ & $43,9( \pm 0,7)$ & $42,1( \pm 0,5)$ \\
VCM $\left(\mu^{3}\right)$ & $90,4( \pm 0,7)$ & $90( \pm 0,7)$ \\
Eritrócitos $\left(\mathrm{x} 10^{6} / \mathrm{mm}^{3}\right)$ & $4,9( \pm 0,1)$ & $4,7( \pm 0,1)$ \\
Leucócitos $\left(\mathrm{mm}^{3}\right)$ & $7556( \pm 404)$ & $7095( \pm 365)$ \\
\hline
\end{tabular}

$\mathrm{EPM}=$ erro padrão da média, ${ }^{*} \mathrm{p}<0,05 ; \mathrm{g} / 100 \mathrm{ml}=$ grama por 100 milimetros; $\%=$ porcentagem; $\mathrm{VCM}=$ volume corpuscular médio.

Conforme podemos observar na Tabela 3 , os valores estão aparentados como médias para homens e mulheres respectivamente.
Os exames laboratoriais bioquímicos como a média da glicemia mostraram uma diferença estatística entre homens 104,2 e mulheres

Tabela 3. Comparação de exames bioquímicos, por sexo, de pacientes com DPOC participantes de um programa de reabilitação pulmonar.

\begin{tabular}{lcc}
\hline \multirow{2}{*}{ Variáveis } & \multicolumn{2}{c}{ Sexo } \\
\cline { 2 - 3 } & Masculino & Feminino \\
\cline { 2 - 3 } & Média $( \pm \mathrm{EPM})$ & Média $( \pm \mathrm{EPM})$ \\
\hline Glicemia $(\mathrm{mg} / \mathrm{dL})$ & $104,2( \pm 4,3)^{\star}$ & $89,8( \pm 2,4)$ \\
CT $(\mathrm{mg} / \mathrm{dL})$ & $187,5( \pm 6,3)$ & $201,3( \pm 7,1)$ \\
HDL $(\mathrm{mg} / \mathrm{dL})$ & $50,8( \pm 2,2)$ & $61( \pm 2,7)$ \\
LDL $(\mathrm{mg} / \mathrm{dL})$ & $113,2( \pm 6,2)$ & $118,3( \pm 6,8)$ \\
TGL $(\mathrm{mg} / \mathrm{dL})$ & $125,5( \pm 11,1)$ & $112( \pm 9,3)$ \\
Ureia $(\mathrm{mg} / \mathrm{dL})$ & $34,6( \pm 1,3)$ & $32,2( \pm 1,8)$ \\
Albumina $(\mathrm{g} / \mathrm{dL})$ & $4,1( \pm 0,1)$ & $4,0( \pm 0,1)$ \\
Creatinina $(\mathrm{mg} / \mathrm{dL})$ & $1,1( \pm 0,04)$ & $0,9( \pm 0,03)$ \\
\hline
\end{tabular}

$\mathrm{EPM}=$ erro padrão da média, ${ }^{*} \mathrm{p}<0,05, \mathrm{mg} / \mathrm{dL}=$ miligrama por decilitros, $\mathrm{g} / \mathrm{dL}=$ gramas por decilitros. 
89,8 (mg/dL). Os valores de médios para o Colesterol Total (CT) foram de 187,5 e 201,3 $\mathrm{mg} / \mathrm{dL}$. Os valores médios de HDL, LDL e Triglicerídeos (TGL) foram respectivamente: 50,8 e $61 \mathrm{mg} / \mathrm{dL} ; 113,2$ e 118,3 mg/dL; 125,5 e $112 \mathrm{mg} / \mathrm{dL}$. A média dos valores encontrados para a ureia foram 34,6 e 32,2 mg/dL. A Albumina e a Creatinina média foram respectivamente, 4,1 e 4,0 mg/dL e 1,1 e 0,9 mg/ dL. Os exames laboratoriais são importantes aliados no controle e acompanhamento da evolução do DPOC.

\section{DISCUSSÃO E CONCLUSÃO}

Em 2020 a DPOC esta projetada para ser a causa de morte de mais de 6 milhões de pessoas anualmente no mundo, tornando assim a terceira causa de morte no mundo o conhecimento da doença tem melhorado muito nos últimos 10 anos. Estudos epidemiológicos têm demonstrado a importância de sabermos também as comorbidades nesses pacientes uma vez que a sua mortalidade pode ser influenciada $(1,12,13,10)$.

Doenças cardíacas e vasculares estão associadas com mortalidade e admissão hospitalar, piora da qualidade de vida e estão frequentemente acompanhadas a outras comorbidades como a diabetes e DPOC. A presença de diabetes em portadores de DPOC pode aumentar a mortalidade associado a cardiopatias, as taxas de internações hospitalares, diminuição da qualidade de vida e a funcionalidade destes pacientes (14). Os mecanismos fisiopatológicos conhecidos nas alterações vasculares de DPOC parecem ser principalmente mediados por disfunção endotelial e coagulopatias. Onde a inflamação sistêmica observada no DPOC parece ter um papel determinante no desenvolvimento de disfunções, pulmonares, sistêmicas e endotelial embora o mecanismo exato ainda é desconhecido. As doenças coronarianas e DPOC são ambas as doenças inflamatórias e envolvem anomalias da coagulação onde a presença da DPOC pode contribuir para o desenvolvimento de doenças cardiovasculares através da dispneia, inflamação sistêmica e stress oxidativo (8). Um estudo recente com 342 portadores de DPOC onde três meses após a primeira exacerbação 64\% destes tinham alterações cardíacas significativas avaliadas por ecografia transtorácica, porém no presente estudo foi encontrado que $17 \%$ dos homens eram cardiopatas em 5,5\% das mulheres (15).

Neste estudo pode-se observar que os valores dos parâmetros bioquímicos avaliados encontram-se dentro da normalidade, exceto para a glicemia que foi significativamente maior nos homens quando comparado com as mulheres e o colesterol que foi maior que o limite desejável para mulheres, embora a média dos valores de glicemia neste grupo permanecem dentro da faixa de pre-diabetes conforme (16). Esta comorbidade já foi identificada por outros estudos, uma preocupação considerando que a prevalência de diabetes em pacientes com DPOC variam entre os estudos 10,3\% no centro de reabilitação (17), 12,6 a 14,5\% (18), 12,2\% com um aumento do risco para fumantes ativos (19) e 18,7\% (20) onde em nosso estudo foi encontrado 14,8 \% dos homens com DM, sendo esta comorbidades a única que tem uma diferença estatisticamente significativa. Um estudo de Coorte demonstrou que pacientes que apresentam DPOC moderado a grave tem aumento no risco de desenvolver Diabetes (OR 1,4 e 1,5 respectivamente) (18). Outra preocupação com pacientes diabéticos é a presença de inflamação sistêmica, que esta associada com disfunção endotelial e pode contribuir em manter o processo inflamatório pulmonar independente do grau de obstrução do fluxo aéreo em portadores de DPOC (21).

Estudos sugerem que DPOC pode ser um fator de risco para o desenvolvimento de diabetes tipo 2 por ser uma doença inflamatória crônica com aumento de interleucinas 
(IL-6, IL-8) e Fator de Necrose Tumoral alfa (TNF $\alpha$ ) e outros mediadores inflamatórios que podem implicar em resistência a insulina (22). Além do comprometimento das alterações inflamatórias pulmonares pacientes diabéticos com DPOC tem alterações musculares periféricas que aceleram o processo de perda de massa muscular, sendo um preditor de mortalidade independente do grau de obstrução do fluxo aéreo (23). Estudos demonstram que o paciente diabético tem comprometimento funcional considerável associado à redução da qualidade de vida, alterações essas bem evidenciadas em portadores de DPOC (24-26).

Embora não necessariamente causalmente associada com a doença, à existência de DPOC podem realmente aumentar o risco de outra doença. Comorbidades podem ocorrer em pacientes em qualquer grau de obstrução da doença e não esta restrita a doença avançada. Entretanto as comorbidades são associadas com aumento da morbidade e mortalidade. A reabilitação pulmonar é uma intervenção não farmacológica com benefícios para pacientes com DPOC e nas suas comorbidades $(1,10,27,28)$.

Em conclusão, os resultados do estudo identificaram o perfil dos pacientes portadores de DPOC onde observa-se que os resultados dos exames bioquímicos e hematológicos, exceto a glicemia e colesterol total para mulheres, estão dentro da normalidade. Contudo, são necessários mais estudos sobre o perfil demográfico, clínico e laboratorial de pacientes portadores de doença pulmonar obstrutiva crônica no Brasil no sentido de caracterizar melhor esta população e proporcionar um tratamento mais individualizado ao paciente de acordo com as características de cada região.

Com base nesse conhecimento, podem-se apontar estratégias de trabalho mais condizentes com a realidade e políticas mais eficazes que busquem estimular uma maior integralidade na assistência aos indivíduos com DPOC, realizando ações de promoção e prevenção, uma vez que as comorbidades associadas a doenças podem ser minimizadas. Sendo fundamental a participação da equipe multidisciplinar e da sistematização da assistência de enfermagem, para traçar um plano de cuidados ao paciente portador de DPOC, bem como o acampamento dos parâmetros clínicos e laboratoriais de forma sistemática.

\section{REFERÊNCIAS}

1. Global Initiative for Chronic Obstructive Lung Disease (GOLD). Global Strategy for the Diagnosis, Management, and Prevention of Chronic Obstructive Pulmonary Disease [Internet]. Bethesda, MD: National Institutes of Health; 2014 [citado 1 mar 2015]. 83 p. Disponivel em: http://goldcopd.org/.

2. Rabahi MF. Epidemiologia da DPOC: Enfrentando Desafios. Pulmão. 2013; 22(2): 4-9.

3. Ike D, Jamami M, Marino DM, Ruas G, Pessoa BV, Di Lorenzo VAP. Efeitos do exercício resistido de membros superiores na força muscular periférica e na capacidade funcional do paciente com DPOC. Fisioter. Mov. 2010; 23(3): 429437.

4. Nici L, Donner C, Wouters E, Zuwallack $\mathrm{R}$, Ambrosino N, Bourbeau J, et al. American Thoracic Society/European Respiratory Society statement on pulmonary rehabilitation. Am J Respir Crit Care Med. 2006; 173(12): 1390-413.

5. Stephens MB, Yew KS. Diagnosis of chronic obstructive pulmonary disease. Am Fam Physician. 2008; 78(1): 87-92.

6. O'Donnell DE. Hyperinflation, dyspnea, and exercise intolerance in chronic obstructive pulmonary disease. Proc Am Thorac Soc. 2006; 3(2): 180-184.

7. Global Initiative for Chronic Obstructive Lung Disease-GOLD. Global Strategy for the Diagnosis, Management and 
Prevention of Chronic Obstructive Lung Disease. NHLBI/ WHO Workshop report. Bethesda, MD: National Institutes of Health; 2006.88 p.

8. Cavaillès A, Brinchault-Rabin G, Dixmier A, Goupil F, Gut-Gobert C, Marchand-Adam S, et al. Comorbidities of COPD. Eur Respir Rev. 2013; 22(130): 454-475.

9. Ferrari R, Tanni ES, Lucheta PA, Faganello MM, Amaral RAF, Godoy I. Preditores do estado de Saúde em pacientes com DPOC de acordo com o gênero. J Bras Pneumol. 2010; 36(1): 37-43.

10. Franssen FME, Rochester, C. Comorbidities in patients with COPD and Pulmonary Rehabilitation: do they matter? Eur Respir Rev. 2014; 23(131): 131-141.

11. Thomsen M, Dahl M, Lange P, Vestbo J. Nordestgaard. Inflammatory biomarkers and comorbidities in chronic obstructive pulmonary disease. Am J Respir Crit Care Med. 2012; 186(10): 982-988.

12. Spruit MA, Singh SJ, Garvey C, ZuWallack R, Nici L, Rochester C, et al. An official American Thoracic Society/ European Respiratory Society statement: key concepts and advances in pulmonary rehabilitation. Am J Respir Crit Care Med. 2013; 188(8): e13-64.

13. Ko FWS, Lim TK, Hancox RJ, Yang IA. Years in review 2013: chronic obstructive pulmonary disease, asthma and airway biology. Respirology. 2014; 19(3): 438447.

14. Pelle AJ, Loerbroks A, Widdershoven JW, Denollet J. Heart failure and comorbid diabetes mellitus or chronic obstructive pulmonary disease. Effects on mood in outpatients. Int J Cardiol. 2012; 154(2): 216-217.

15. Freixa X, Portillo K, Paré C, García Aymerich J, Gómez FP, Benet M, et al. Echocardiographic abnormalities in patients with COPD at their first hospital admission. Eur Respir J. 2013; 41(4): 784-791.

16. Sociedade Brasileira de Diabetes. Dire- trizes da Sociedade Brasileira de Diabetes 2013/2014 [Internet]. São Paulo: AC Farmacêutica; 2014 [citado em 25 Sep 2014]. Disponível em: http://www.sgc.goias.gov. br/upload/arquivos/2014-05/diretrizes-sbd-2014.pdf

17. Cristafulli E, Costi S, Luppi F, Cirelli G, Cilione C, Coletti O, et al. Role of comorbidities in a Cohort of patients with COPD undergoing pulmonar rehabilitation. Thorax. 2008; 63(6): 487-92.

18. Mannino DM, Thorn D, Swensen A, Holguin F. Prevalence and outcomes of diabetes, hypertension and cardiovascular disease in COPD. Eur Respir J. 2008; 32(4): 962-969.

19. Feary JR, Rodrigues LC, Smith CJ, Hubbard RB, Gilson GE. Prevalence of major comorbidities in subjects with COPD and incidence of myocardial infarction and stroke: a comprehensive analysis using data from primary care. Thorax. 2010; 65(11): 956-962.

20. Cazzola M, Bettoncelli G, Sessa E, Cricelli C, Biscione G. Prevalence of comorbidities in patients with chronic obstructive pulmonary disease. Respiration. 2010; 80(2): 112-119.

21. Goldman MD. Lung dysfunction in diabetes. Diabetes Care. 2003; 26(6): 19151918.

22. Rana JS, Mittleman MA, Sheikh J, HU FB, Manson JE, Colditz GA, et al. Chronic obstructive pulmonary disease, asthma, and risk of type 2 diabetes in women. $\mathrm{Di}$ abetes Care. 2004; 27(10): 2478-2484.

23. Ferrucci L, Studenski S. Diabetes, muscles and the myth of Ulysses' bow. Diabetes Care. 2009; 32(11): 2136-2137.

24. Daudey L, Peters JB, Molema J, Dekhuijzen PN, Prins JB, Heijdra YF, et al. Health status in COPD cannot be measured by the St George's Respiratory Questionnaire alone: an evaluation of the underlying concepts of this questionnaire. Respir Res. 2010; 11: 98.

25. Hernandes NA, Teixeira DC, Probst VS, 
Brunetto AF, Ramos EC, Pitta F. Perfil do nível de atividade física na vida diária de pacientes portadores de DPOC no Brasil. J Bras Pneumol. 2009; 35(10): 949-956.

26. Sinclair AJ, Conroy SP, Bayer AJ. Impact of diabetes on physical function in older people. Diabetes Care. 2008; 31(2): 233235.

27. Koeskela J, Kilpeläinen M, Kupiainen $H$, Mazur W, Sintonen H, Boezen M, et al.
Co-morbidities are the key nominators of the health related quality of life in mild and moderate COPD. BMC Pulm Med. 2014; 14: 102.

28. Yaksic MS, Tojo M, Culier A, Stelmach R. Perfil de uma população brasileira com doença pulmonar obstrutiva crônica grave. J Bras Pneumol. 2003; 29(2): 6468. 